\title{
An Experimental Research on GPS Based Boundary Intruding Boat Monitoring System
}

\author{
R. Puviarasi, M. Mageshwaran, Mritha Ramalingam, S.R.Boselin Prabhu
}

\begin{abstract}
Boundary detection and alert system is a straightforward and effective idea, which utilizes Internet of Things technology. By utilizing this framework border monitoring is $100 \%$ protected and secure. It naturally alarms the intruder when the vehicle goes over the specific range in borders. This is finished by a sensor called Global positioning system (GPS). It detects the current position of the vehicle and switch on the caution framework naturally. In this anticipate, no need of manual operations like on time and off time setting. GPS and IoT are the fundamental segments of the task. The resistances of the alert system changes as per the distance between the current position of the vehicle and the border get decreased or increased.

Keywords: GPS, IoT, networking, c programing
\end{abstract}

\section{INTRODUCTION}

Checking ecological limits has been a fascinating subject for a long time because of logical and open security applications. From Tamil Nadu around 18,000 vessels of various types lead fishing along the India-Sri Lanka oceanic outskirt [8]. Most of the coastal area villages rely on the fishing occupation only, but crossing the borders in the ocean for any reason is treated as a national offence. Because of ignorance about as far as possible, the fishermen used to cross the oceanic fringes. When they cross the fringe, they captured or murdered by the applicable naval force and they are being stole and their water crafts are being caught by the area nations waterfront watches. Under such circumstance the lives of fishermen keeps on being in threat $[6,7]$. Furthermore, it has turned out to be one of the central points for misfortune in people just as their nation monetary. So to handle this situation, the awareness has to be taught to the fishermen that they have to remain inside the Indian border and not to enter the Sri Lankan border range. The technological framework should be the wellsuited decision for settling the border limit crossing issue. To dispense with such troubles a framework has been produced which encourages the fishermen to know about intersection the fringe. We present framework called advanced GPS based boundary detection and alert system using IoT and GPS technology. This paper accompanies a reliable answer for this issue and shields the Indian fishermen from risky circumstance and being crossing the sea limit and spares their life and enhances the wellbeing of

Revised Manuscript Received on July 18, 2019.

Dr. R. Puviarasi, Department of ECE, Saveetha School of Engineering, Saveetha Institute of Medical and Technical Sciences, Chennai 602105

M. Mageshwaran, Department of ECE, Saveetha School of Engineering, Saveetha Institute of Medical and Technical Sciences, Chennai 602105.

Mritha Ramalingam, Faculty of Computer Systems and Software Engineering, Universiti Malaysia Pahang, Kuantan, Malaysia.

Dr.S.R.Boselin Prabhu, Associate Professor, Department of Electronics and Communication Engineering, Surya Engineering College, Mettukadai, India. fishermen. The framework is planned by utilizing GPS and IoT. A GPS course gadget is a gadget that unequivocally finds current location by getting GPS coordinates from the satellites. This gadget collects the location coordinates continuously and examines where the vessel is currently moving, and it is used locate the point at where the vessel crossed the boundary. This is a critical issue related to security and creates inconvenience between the fishermen and coastguards. GPS based boundary detection and alert system is a framework which helps the fishermen by representing the border using an alert system connected with a global positioning system (GPS). The GPS collects the present scope and longitude esteems and send the coordinates to the microcontroller unit. As soon as the controller receives the current coordinates it locates the vessel with respect to the border, by contrasting the received coordinates and qualities with the predefined esteem. At the equilibrium point of both coordinates, this system intimates the fishermen that they are going to achieve the border outskirt. Remembering about existences of Indian fishermen, this gadget has been made to help them not to move past Indian fringe. It encourages the fishermen not to go a far distance of outskirt. On the off chance that the fishermen disregard the outskirt and enters the prohibited area, an alert (risk flag) is produced demonstrating that the fishermen have violated the boundary of the nation.

A GPS-based remote impromptu system is a device which is used for Checking, inquiry, and salvage marine applications in Vietnam. This system's steering convention and calculation are assessed utilizing System Test system 2 programming. The outcomes demonstrate a triumph amount of bundles transferring more than $85 \%$. It demonstrates the incredible capability of proposed idea. Catchphrases specially appointed system, marine observing and seeking, Worldwide Situating Framework (GPS).

The proposed framework was used for identifying the nation's border through the predetermined longitude and scope of the boundary, between Sri Lanka and India as well as everywhere throughout the world. The specific layer level for example fringe can be predefined and this can be put away in PIC microcontroller memory. The present esteem is contrasted and predefined values and if the qualities are same, quickly the specific task will be done for example the PIC microcontroller offers guidance to the caution to ringer. It likewise utilizes a data transmitter to send the message to the land, where the vessel is screened by the marine patrol [5]. The device intimate the message to the fishermen and for the marine patrol situated in the landside. This process

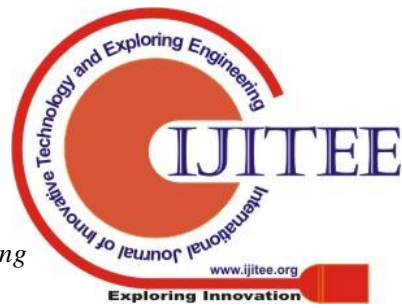


will be useful for the marine patrols to guide the coastguard to the intruded area and to rescue the boat and fishermen [1]. This framework was utilized for distinguish the oceanic limit for the two nations to reduce the violations in the ocean. This is for making the fishermen to be aware of the boundaries where the breakpoints are not visible to the naked eye. The proposed framework utilizes a GPS sensor which collects latitude and longitude coordinates from the satellites and identifies the present position of the vessel. And compares the obtained coordinates with definitely known latitude and longitude coordinates of the boundary, the PIC microcontroller calculates the difference between both the latitude and longitude coordinates. When the difference between them is getting reduced, at the equilibrium position the microcontroller alerts the fishermen by intimating the alert system. It additionally sends a message to the marine patrol in the landside by using a data transmitter. For the marine patrols in land can guide the coastguard to help the fishermen. Along these lines this device is used to save the life of the fishermen or diminishes the attacks caused due to the Sri Lankan patrols [2].

The framework is for the most part for anglers are utilized to identify the sea limit between the two nations. This for the most part happens when angler crosses oceanic fringe of neighboring nation as he doesn't know about the cutoff points in ocean. The proposed framework utilizes a sensor called global positioning system to collect the location coordinates from the satellites and locate the current position of vessel in the ocean. It receives the latitude and longitude coordinates of sensor to locate the present position of the device which is located in the vessel, when it reaches the corresponding location it intimates the fishermen that he has crossed the limit of their countries border by giving an alert, vibrate and warning. The message will be sent to server segment about the intrusion of the boundary, and the angler will obtain the caution outwardly. This process will help the angler close to the outskirt area easily [3]. The speaker started with the perception that India imparts sea limits to the same number of nations as it shares land fringes. Authoritatively, there is no oceanic limit issue among India and Sri Lanka. The connection between both the nations had been customarily sincere. The two nations are occupied with wide running regions. The connection between the two fills in as a model of good neighborly communications [4].

The remaining contents of the paper are sorted out as follows. In $1^{\text {st }}$ section, the GPS system and monitoring systems are discussed. The overall framework of proposed monitoring system is presented in $2^{\text {nd }}$ section. $3^{\text {rd }}$ section discusses about the implementation of proposed framework. In $4^{\text {th }}$ section, the result of the proposed system is discussed. Finally, the framework is concluded in $5^{\text {th }}$ section.

\section{GPS BASED BOUNDARY INTRUDING BOAT MONITORING SYSTEM}

Fig. 1 describes the fundamental blocks of the GPS based boundary intruding boat monitoring system. Each block performs the various operations in the framework.

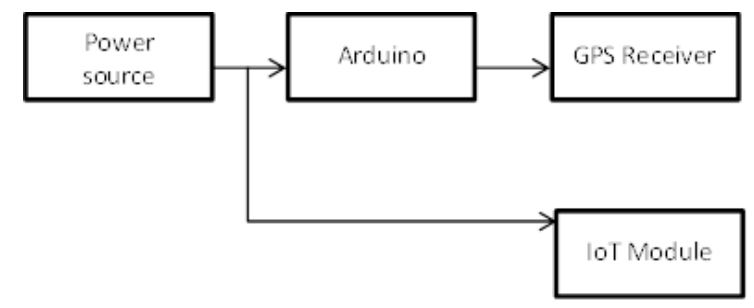

Fig. 1. Block diagram of GPS based boundary intruding boat monitoring system

Power source is the component that provides enough power to run the GPS module and IoT module. The required source could be a battery or regulated power supply. The power required running the IoT module is 3 volt, and the power required to run the GPS module is 5 volt. The GPS and IoT modules require Dc power supply.

\section{Arduino}

Arduino alludes to an open-source gadgets stage or board and the product used to program it. Arduino is intended to make gadgets progressively open in making intelligent articles or conditions. In this framework the Arduino is used only to provide the power supply to the GPS receiver.

\section{GPS Module}

Global Positioning System depends on satellite route innovation. A GPS Recipient gives the exact area of an article as far as scope and longitude. Exact time count as for GMT should likewise be possible by utilizing GPS. GPS gives a great deal of topographical data for a specific article like its scope, longitude, heading of movement, GMT and so on. This data are amassed in specific string positions which are to be decoded by GPS modems. The GPS receiver receives the current position coordinates continuously from the satellites and transmits the received coordinates to the IoT module.

\section{IoT Module}

An IoT module is a little electronic gadget implanted in articles, machines and things that interface with remote systems and sends and gets information. Once in a while alluded to as a "radio chip", the IoT module contains a similar innovation and information circuits found in cell phones yet without highlights like a showcase or keypad. The IoT module provides always on connectivity. It transfers data automatically without any instructions needed. It is developed for extreme durability and longevity and for continuous operations. The IoT module compares the GPS coordinates with the predefined boundary value and switches the alert system if the boundary is violated.

\section{METHODOLOGY}

The framework Unit introduced in each vessel ceaselessly recovers its GPS organizes and ascertains the separation between the pontoon and the put away estimations of the sea fringe. At the point when the separation between the pontoon and the outskirt is beneath the reference esteem, an alert is activated in the watercraft. Also, the message is sent 
to the mail id by the IoT module. The framework comprises of two noteworthy units which arrange among one another effectively. They are GPS module and IoT module. The framework Unit introduced in each vessel ceaselessly examines the GPS area of the vessel. It compares the separation between the vessel and border and if the separation is underneath the reference esteem, the IoT module triggers the alert which is message notice in the display. The IoT also sends the alert message to the predefined email id about the intrusion of the boundary. The email id could be connected to the coast guard patrol service so that the coastguard can accommodate the fishermen safely back inside the boundary.

Algorithm

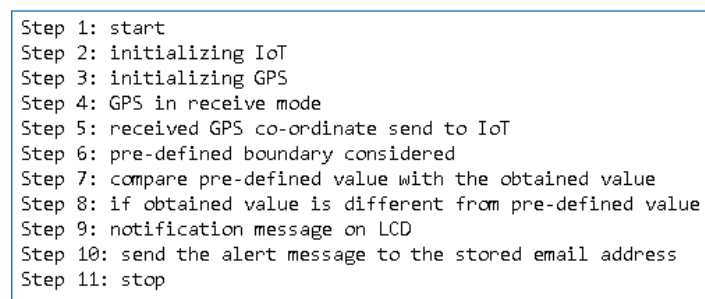

Fig. 2. Algorithm for programing the module

Fig 2 describes the algorithm for programming the IoT module using the $\mathrm{C}$ programming language.

\section{RESULT ANALYSIS}

We have developed a framework which reduces such issues and saves the lives and economic issues of the fishermen. The system detects the nation's boundary and alerts the vessel and the guardian if the intrusion occurs.

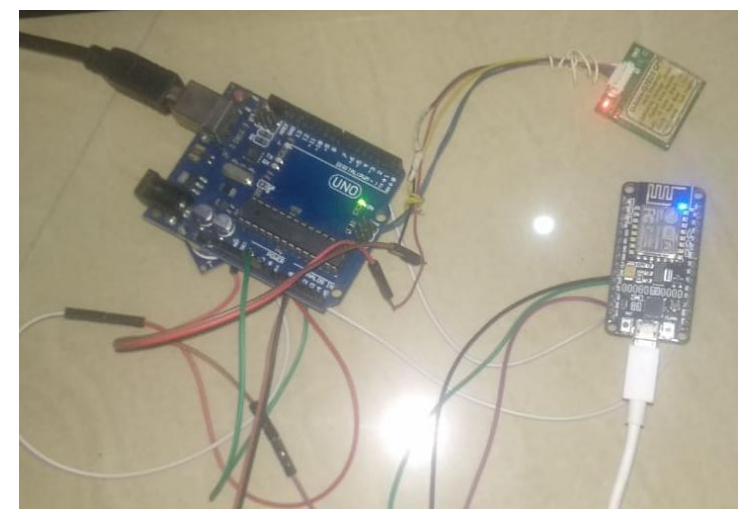

\section{Fig. 3. Experimental setup of GPS Based boundary intruding boat monitoring system}

Fig 3 shows the experimental setup of the GPS based boundary intruding boat monitoring system. It contains all the components included in the block diagram of this project. The GPS receiver, IoT module and the Arduino are connected together to perform the designed operation.

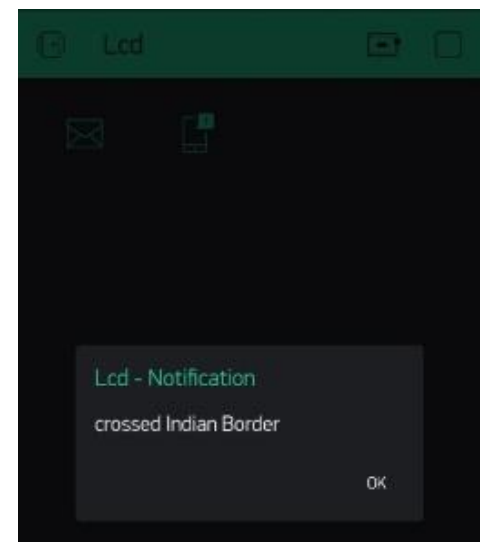

Fig. 4. LCD notification in the boat unit

Fig 4 indicates the received notification on the LCD screen on the boat unit about the intrusion of the boundary

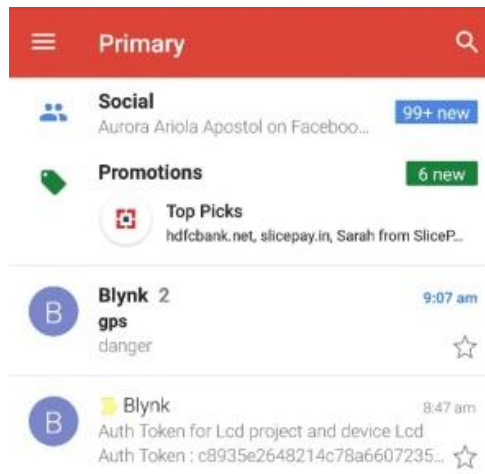

Fig. 5. Message alert through the email

Fig 5 indicates the received notification in the form of message through the email id, using the internetworking technology.

\section{CONCLUSION}

In this paper, a GPS based boundary detection system is proposed for avoiding the intrusion of boundaries. By using this system the borders will be protected and secured effectively. In this system, the Global Positioning System (GPS) device is used with the internet of things (IoT) technology to identify the current location, and intimate the fishermen automatically using the alarm. The proposed system is implemented using major components of GPS and IoT. The hardware results are analyzed and explained. In future the communication process can be made better by using advanced technology like LIFI etc.

\section{REFERENCES}

1. Balaji, V., Vivekanadan, M., Anaga Suba Raja, S.: Location based system using gps-fishermen sms alert system. In: NCET, 4. 2319-8753, 152-157 (2015)

2. Mahesh, S., Karthik Eshwar E.: Design of maritime boundary identification system and fishermen patrol system. In: IEEE, 3. 916-1503, 1-4 (2014)

3. Yuvaraj, E., Arunvijay, D.: Design of border alert system for fishermen using gps. In: IJSRT, 2. 2321-2543, 67-70 (2014) 
4. Manoharan, N.: Indias Maritime Neighborhood: Issues and Option - Sri Lankan Case. In: IJECT, 6. 2121-2653, 1-5 (2014)

5. Montgomery, D.R.: International Fisheries Enforcement Management Using Wide Swath SAR. In: JHATD, 21. 503951, 105-112 (2011)

6. Ranjith, S., Shreyas, Pradeep Kumar, K., Karthik, R.: Automatic Border Alert System for Fishermen using GPS and GSM Techniques. In: Indonesian Journal of Electrical Engineering and Computer Science, Vol. 7, pp. $84-89$ (2017)

7. Sivaramaganesh, M., Ramya, M., Gowtham, V., Bharathi, T., Jeevitha, G.: Implementation of Maritime Border Alert System. In: International journal of innovative research in electrical, electronics, instrumentation and control engineering, Vol. 2 (2014)

8. Reynolds, J.C., Denaro, R.P., Kalafus, R.M.: GPS-based vessel position monitoring and display system. In: IEEE Aerospace and Electronic Systems Magazine (1990). 